\title{
Bifurcations of equilibria of a non-linear age structured model
}

\author{
J. Z. Farkas
}




\title{
BIFURCATIONS OF EQUILIBRIA OF A NON-LINEAR AGE STRUCTURED MODEL
}

\author{
J. Z. FARKAS
}

[Received: October 29, 2003]

\begin{abstract}
In [6], M. E. Gurtin and R. C. MacCamy investigated a non-linear agestructured population dynamical model, which served as one of the basic non-linear population dynamical models in the last three decades. They described a characteristic equation but they did not use it to discuss stability of equilibria of the system in certain special cases. In a recent paper [4] M. Farkas deduced a characteristic equation in another form. This characteristic equation enabled us to prove results about the stability of stationary age distributions of the system, [3]. In the present paper we are going to investigate how equilibria arise and change their stability as a basic parameter of the system varies.
\end{abstract}

Mathematics Subject Classification: 70K20, 92B05

Keywords: stability of equilibria, bifurcation

\section{INTRODUCTION}

We consider the following model equation:

$$
p_{a}^{\prime}(a, t)+p_{t}^{\prime}(a, t)=-\mu(a, P(t)) p(a, t),
$$

where $p(a, t)$ denotes the density of population of age $a,(0 \leq a \leq m<\infty)$ at time $t, \mu(a, P(t))$ denotes the mortality of individuals of age $a$ at the population quantity $P(t)$, which quantity at time $t$ is given by the relation

$$
P(t)=\int_{0}^{m} p(a, t) d a .
$$

The number of newborns at time $t$ is given by the equation

$$
p(0, t)=\int_{0}^{m} \beta(a, P(t)) p(a, t) d a,
$$

with the fertility function $\beta(a, P(t))$. Finally, we assume a given age distribution at time $t=0$,

$$
p(a, 0):=p_{0}(a) .
$$

The author was supported in part by the Hungarian NFSR, Grant No. T046124. 
The functions $\beta(\cdot, \cdot), \mu(\cdot, \cdot) \in C^{1}$ and, naturally, they are non-negative. We assume a finite maximal age denoted by $m$. This assumption does not make any restriction in a biological sense setting $m$ as a "very large number", but lets us avoid some mathematical problems.

The dynamics of the system (1.1)-(1.4) is determined by the vital rates $\beta(a, P(t))$ and $\mu(a, P(t))$ depending on the age $a$ and on the total population quantity $P(t)$. Many papers deal with models where $P(t)$ is replaced by a weighted size function $S(t)=\int \gamma(a) p(a, t) d a$, or more generally a finite number of such functions $S_{i}(t)=\int \gamma_{i} p(a, t) d a, i=1 . . n,[\mathbf{2}, 7]$.

Theorem 1 (M. E. Gurtin \& R. C. MacCamy [6]). Any stationary solution $p_{1}(a)$ of the system is determined uniquely by the root $P_{1}$ of the following equation

$$
R(P)=\int_{0}^{m} \beta(a, P) \pi(a, P) d a=1 .
$$

Here $\pi(a, P)=e^{-\int_{0}^{a} \mu(s, P) d s}$ is the probability for any individual to reach the age $a$, and $R(P)$ is the net reproduction number. Then

$$
p_{1}(a)=\frac{P_{1} \pi\left(a, P_{1}\right)}{\int_{0}^{m} \pi\left(a, P_{1}\right) d a} .
$$

Definition 1. The stationary solution $p_{1}(a)$ of the system is said to be asymptotically stable if there exists a $\delta>0$ such that whenever $\left|p(a, 0)-p_{1}(a)\right|_{L^{1}}<\delta$, one has $\lim _{t \rightarrow \infty}\left|p(a, t)-p_{1}(a)\right|_{L^{1}}=0$.

The stability of the trivial equilibrium is mainly characterised by the following theorem.

Theorem 2 (M. Iannelli [7]). If $R(0)<1$, then the trivial equilibrium $p_{1}(a) \equiv 0$ is asymptotically stable, and if $R(0)>1$, then it is unstable.

As we mentioned earlier in [3] we proved some results about the stability of stationary solutions. One of them characterises the stability in a case when the vital rates assume a special form, namely,

$$
\mu(a, P(t))=m(a), \quad \beta(a, P(t))=b(a) f(P(t)),
$$

a factorisation property for $\beta(\cdot, \cdot)$.

Theorem 3 (J. Z. Farkas [3]). If $R^{\prime}\left(P_{1}\right)<0$, then the stationary solution corresponding to the population quantity $P_{1}=\int_{0}^{m} p_{1}(a) d a$ is asymptotically stable, and if $R^{\prime}\left(P_{1}\right)>0$, then it is unstable.

In [1], J. M. Cushing investigated a general non-linear McKendrick model. $\mathrm{He}$ proved that at the critical value of the bifurcating parameter $n=R(0)=1$ two positive equilibria arise from the trivial equilibrium and he studied stability both for the trivial and the positive equilibria in a small neighbourhood of the critical parameter value 
by means of the principle of linearised stability. He used implicit function techniques under smoothness conditions on the vital rates as functionals of age and population density.

In the recent paper we are going to investigate a family of systems for which a "global" theorem proves the linear stability or instability of equilibria. Saddle-node bifurcation "far" from the trivial equilibrium and transcritical bifurcation near the trivial equilibrium are shown.

\section{Bifurcations of EQUiLibria}

Now consider a family of systems with the following vital rates

$$
\mu(a, P)=m(a), \quad \beta_{c}(a, P)=b(a) f_{c}(P), \quad f_{c}(P)=\frac{1}{P+1}+c P
$$

with the bifurcation parameter $c>0, m, \beta_{c} \in C^{1}$. Setting $K=\int_{0}^{m} b(a) \pi(a) d a$, we get the equation

$$
K f_{c}\left(P_{1}\right)-1=\frac{c K P_{1}^{2}+c K P_{1}+1}{P_{1}+1}-1=0
$$

for the population quantity $P_{1}$. Observe that now $R(0)=K$ holds.

From the equation

$$
c K P_{1}^{2}+(c K-1) P_{1}+K-1=0
$$

we get

$$
P_{1}^{1,2}=\frac{(1-c K) \pm \sqrt{c^{2} K^{2}-4 c K^{2}+2 c K+1}}{2 c K}
$$

and we seek for a positive equilibrium.

\subsection{The CASE $K>1$}

In (2.4), the relation

$$
c^{2} K^{2}-4 c K^{2}+2 c K+1>0
$$

holds if

or

$$
0<c<2-\frac{1}{K}-2 \sqrt{1-\frac{1}{K}}
$$

$$
c>2-\frac{1}{K}+2 \sqrt{1-\frac{1}{K}}
$$

We first consider the interval $0<c<2-\frac{1}{K}-2 \sqrt{1-\frac{1}{K}}$, and, in view of the inequality $\frac{1}{K}>2-\frac{1}{K}-2 \sqrt{1-\frac{1}{K}}$, we now have $1-c K>0$. The inequality $1-c K>$ $\sqrt{c^{2} K^{2}-4 c K^{2}+2 c K+1}$ shows that, in this interval of values of the parameter $c$, there exist exactly two non-trivial equilibria. 
At the parameter value $c=2-\frac{1}{K}-2 \sqrt{1-\frac{1}{K}}$ we have $c^{2} K^{2}-4 c K^{2}+2 c K+1=0$ and $1-c K>0$, so, at this value of $c$, there exists only one positive equilibrium with total population quantity $P_{1}=\frac{1-K+K \sqrt{1-\frac{1}{K}}}{2 K-1-2 K \sqrt{1-\frac{1}{K}}}$.

For $2-\frac{1}{K}-2 \sqrt{1-\frac{1}{K}}<c<2-\frac{1}{K}+2 \sqrt{1-\frac{1}{K}}$ there are not any positive equilibria, and this holds for $c>2-\frac{1}{K}+2 \sqrt{1-\frac{1}{K}}$ because $1-c K<0$ and $c K-1>\sqrt{c^{2} K^{2}-4 c K^{2}+2 c K+1}$.

Now we are going to examine the stability of the equilibria. We first consider the positive equilibrium at the parameter value $c=2-\frac{1}{K}-2 \sqrt{1-\frac{1}{K}}$ with total population quantity $P_{1}=\frac{1-c K}{2 c K}=\frac{1-K+K \sqrt{1-\frac{1}{K}}}{2 K-1+2 K \sqrt{1-\frac{1}{K}}}$. We have

$$
f_{c}^{\prime}(P)=c-\frac{1}{P^{2}+2 P+1},
$$

and an elementary calculation gives

$$
f_{c}^{\prime}\left(P_{1}\right)=0 .
$$

It is easy to show that for $P>P_{1}$ we have $f_{c}^{\prime}(P)>0$ and for $P<P_{1}$ we get $f_{c}^{\prime}(P)<$ 0 . Applying Theorem 3 we get that one of the positive equilibria is asymptotically stable and the other one is unstable at the parameter interval $0<c<2-\frac{1}{K}-$ $2 \sqrt{1-\frac{1}{K}}$. The trivial equilibrium is unstable by Theorem 2 because $R(0)=K>1$. Summarising, we have the following

Theorem 4 (Saddle-node bifurcation). For $c>2-\frac{1}{K}-2 \sqrt{1-\frac{1}{K}}$, there is no positive equilibrium, for $c_{0}=2-\frac{1}{K}-2 \sqrt{1-\frac{1}{K}}$ we have one positive equilibrium. For $c<c_{0}$, there are two curves of positive equilibria in a neighbourhood of

$$
(P, c)=\left(\frac{1-K+K \sqrt{1-\frac{1}{K}}}{2 K-1-2 K \sqrt{1-\frac{1}{K}}}, c_{0}\right) .
$$

The upper equilibria are unstable and the lower equilibria are asymptotically stable.

\subsection{The CASE $K<1$}

Now $c^{2} K^{2}-4 c K^{2}+2 c K+1>0$ for every $c>0$. If $0<c<\frac{1}{K}$, then $1-c K>0$ and in view of the relation $1-c K<\sqrt{c^{2} K^{2}-4 c K^{2}+2 c K+1}$, we get one positive non-trivial equilibrium with total population quantity

$$
P_{1}=\frac{1-c K+\sqrt{c^{2} K^{2}-4 c K^{2}+2 c K+1}}{2 c K} .
$$


For $c \geq \frac{1}{K}$ we have $1-c K \leq 0$ and $1-c K+\sqrt{c^{2} K^{2}-4 c K^{2}+2 c K+1} \leq 0$, so there are no positive equilibria.

The positive equilibrium above tends to zero if $c$ tends to $\frac{1}{K}$ and tends to infinity if $c$ tends to 0 . For this positive equilibrium,

$$
f_{c}^{\prime}\left(P_{1}\right)=c-\frac{1}{P_{1}^{2}+2 P_{1}+1}>0
$$

and thus, it is unstable. The trivial equilibrium is asymptotically stable because $R(0)=K<1$.

Theorem 5 (Transcritical bifurcation). There are no positive equilibria for $c \geq \frac{1}{K}$ and, for $c<\frac{1}{K}$, there is a curve of equilibria which is unstable, and the trivial equilibrium is stable.

\subsection{The CASE $K=1$}

Now we have

$$
P^{1,2}=\frac{(1-c) \pm \sqrt{c^{2}-2 c+1}}{2 c},
$$

whence $P^{1}=0$ and $P^{2}=\frac{1}{c}-1$ so for $c<1$ there exists a non-trivial equilibrium. We have

$$
f_{c}^{\prime}\left(P^{2}\right)=c-\frac{1}{\left(\frac{1-c}{c}\right)^{2}+2 \frac{1-c}{c}+1}=c-c^{2}>0
$$

the positive equilibrium is unstable.

For the trivial equilibrium $R(0)=K=1$; but $f_{c}^{\prime}(0)=c-1$ means that for small $P$ we have $R(P)>1$ if $c>1$ and $R(P)<1$ for $c<1$. This means that the trivial equilibrium is stable for $c<1$ and it is unstable for $c>1$.

Theorem 6 (Transcritical bifurcation). For $c \geq 1$ we have only the trivial equilibrium, for $c<1$ a curve of positive unstable equilibria emerges.

\section{Discussion}

In this paper, we have considered the well-known age-structured model of Gurtin an MacCamy in a special case where the mortality of the individuals is age-dependent only and the fertility function is separable in the variables $a$ of age and $P$ of total population quantity. In that case, Theorem 3 establishes the stability of the positive equilibrium, while the stability of the trivial equilibrium depends on the constant

$$
K=\int_{0}^{m} b(a) e^{-\int_{0}^{a} m(s) d s} d a=R(0)
$$

in Theorem 2. We showed that a degenerate transcritical bifurcation occurs, that is, for $K \leq 1$, a curve of positive unstable equilibria bifurcates from the trivial one at the critical parameter value $c_{0}=\frac{1}{K}$ and as $c$ tends to 0 the total population quantity at the 
equilibrium tends to infinity. For $K>1$, we have showed that a saddle-node bifurcation occurs at the critical parameter value $c_{0}=2-\frac{1}{K}-2 \sqrt{1-\frac{1}{K}}$. For $c<c_{0}$, two curves of positive equilibria appear, from which one contains unstable equilibrium points with total population quantity tending to infinity, and the other curve consists of positive stable equilibrium points.

\section{ACKNOWLedgement}

The author is thankful to Prof. Miklós Farkas for his helpful suggestions.

\section{REFERENCES}

[1] Cushing, J. M.: Existence and stability of equilibria in age-structured population dynamics, Journal of Mathematical Biology, 20 (1984), 259-276.

[2] Cushing, J. M.: An Introduction to Structured Population Dynamics, SIAM, Philadelphia, PA 1998.

[3] Farkas, J. Z.: Stability conditions for the non-linear McKendrick equations, to appear in Applied Mathematics and Computations.

[4] Farkas, M.: On the stability of stationary age distributions, Applied Mathematics and Computation, 131 (2002), No. 1, 107-123.

[5] Farkas, M.: Dynamical Models in Biology Academic Press, 2001.

[6] Gurtin, M. E. and MacCamy, R. C.: Non-linear age-dependent populations dynamics, Arch. Rat. Mech. Anal., 54 (1974), 281-300.

[7] Iannelu, M.: Mathematical Theory of Age-Structured Population Dynamics, Giardini Editori, Pisa 1994.

\section{Author's Address}

\section{J. Z. Farkas:}

Budapest University of Technology and Economics, Department of Differential Equations, BuDAPEST, H-1521, HUNGARY

E-mail address: farkas@math. bme.hu 